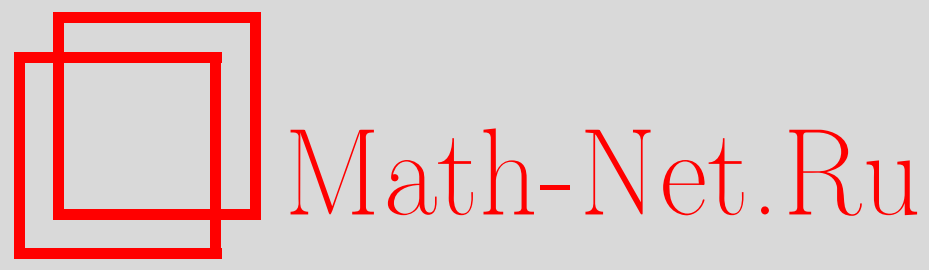

В. А. Гейлер, М. М. Сенаторов, Периодические потенциалы, для которых все лакуны ненулевые, Функи. анализ и его прил., 1997, том 31, выпуск $1,67-70$

DOI: https://doi.org/10.4213/faa446

Использование Общероссийского математического портала MathNet.Ru подразумевает, что вы прочитали и согласны с пользовательским соглашением

http://www.mathnet.ru/rus/agreement

Параметры загрузки:

IP : 35.173 .219 .12

26 апреля 2023 г., 13:39:48

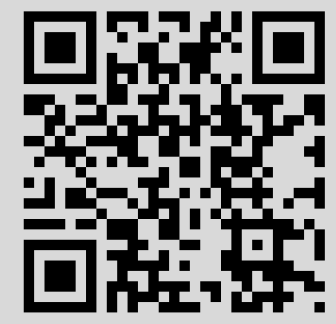




\title{
Периодические потенциалы, для которых все лакуны ненулевые
}

\author{
(C) 1997. В. А. ГЕЙЛЕР, М. М. СЕНАТоров
}

Для $2 T$-периодической функции $U(x)$, суммируемой с квадратом на отрезке $[-T, T]$, периодический оператор Шрёдингера (оператор Хилла) $H$ определяется как замыкание в пространстве $L^{2}(\mathbb{R})$ симметричного оператора, заданного в $C_{0}^{\infty}(\mathbb{R})$ дифференциальным выражением $l[y]=-y^{\prime \prime}+U(x) y$. Пусть $\widetilde{\lambda}_{0}, \lambda_{2}, \widetilde{\lambda}_{2}, \ldots$ - собственные значения периодической $\left(y(-T)-y(T)=y^{\prime}(-T)-\right.$ $\left.y^{\prime}(T)=0\right)$, а $\lambda_{1}, \widetilde{\lambda}_{1}, \lambda_{3}, \widetilde{\lambda}_{3}, \ldots$ - собственные значения антипериодической $\left(y(-T)+y(T)=y^{\prime}(-T)+y^{\prime}(T)=0\right)$ краевых задач, порождаемых на отрезке $[-T, T]$ операцией $l$. Оператор $H$ самосопряжен, его спектр непрерывен и состоит из последовательности сегментов $\left[\widetilde{\lambda}_{0}, \lambda_{1}\right],\left[\widetilde{\lambda}_{1}, \lambda_{2}\right], \ldots,\left[\widetilde{\lambda}_{k-1}, \lambda_{k}\right], \ldots$, разделенных лакунами $\left(-\infty, \widetilde{\lambda}_{0}\right),\left(\lambda_{1}, \widetilde{\lambda}_{1}\right), \ldots,\left(\lambda_{k}, \widetilde{\lambda}_{k}\right), \ldots$ Некоторые лакуны (или даже все, кроме первой) могут оказаться вырожденными [1]. В ряде работ изучалась задача восстановления потенциала $U$ по спектру оператора $H$ (см. [2-4] и др.). В частности, из результатов работы [3] вытекает, что для потенциала $U$ «общего положения» (множество второй категории в $L^{2}(-T, T)$ ) все лакуны невырожденные. Тем не менее конкретных примеров потенциалов такого вида известно довольно мало. Наиболее известны из них $U(x)=\cos x$, приводящий к оператору Матье, а также (разрывный) потенциал Кронига-Пенни, предельный случай которого (гребенка Дирака) используется во многих работах по физике твердого тела [1]. Цель заметки - дать простую конструкцию, позволяющую получить широкий класс непрерывных периодических потенщиалов, для которых все лакуны ненулевые.

Обозначим через $\mu_{k}(T)$ (соответственно $\nu_{k}(T)$ ), где $k=0,1, \ldots$, упорядоченную последовательность собственных чисел задачи Дирихле (Неймана) для оператора Штурма-Лиувилля, заданного дифференциальным выражением $l$ на отрезке $[-T, T]$. Хорошо известно, что при любом $k \in \mathbb{N}$ выполнены неравенства $\nu_{k}(T)<\mu_{k}(T)<\nu_{k+2}(T)$, причем $\mu_{k}$ и $\nu_{k+1}$ лежат в $\left[\lambda_{k+1}, \widetilde{\lambda}_{k+1}\right][5]$. Основными результатами заметки являются следующая теорема и ее следствие.

ТеОРемА. Пусть 2T-периодическая непостоянная функция $U$ допускает продолжение с отрезка $[-T, T]$ до вещественно-аналитической четной выпуклой функции $V$ на всей числовой прямой. Тогда $\mu_{k}(T)<\nu_{k+1}(T)$ для всех $k \in \mathbb{N}$.

СлЕДСТВИЕ. При выполнении условий теоремы в спектре оператора $H$ все лакуны невырожденные.

Доказательство теоремы опирается на следующую лемму.

Лемма. Пусть $V$ - непостоянная выпуклая четная функция класса $C^{(2)}(-1,1)$, и пусть $\left(\mu_{k}\right)$ (соответственно $\left.\left(\nu_{k}\right)\right), k=0,1, \ldots$, - упорядо-

\footnotetext{
* Работа выполнена при финансовой поддержке РФФИ, грант №96-01-00439.
} 
ченнал последовательность собственных чисел задачи Дирихле (Неймана) для оператора Штурма-Лиувилля, заданного дифференииальным выражением $L=-d^{2} / d x^{2}+V$ на отрезке $[-1,1]$. Если $V(1)<\nu_{k+1}$ при некотором $k \in \mathbb{N}$, mo $\mu_{k}<\nu_{k+1}$.

ДоКАЗАТЕЛЬСТво. Положим $E=\nu_{k+1}$ и рассмотрим произвольное ненулевое решение $\varphi$ уравнения $L \varphi=E \varphi$. Тогда функция $u(x)=\varphi^{\prime}(x)$ удовлетворяет уравнению

$$
u^{\prime \prime}(x)+\frac{V^{\prime}(x)}{E-V(x)} u^{\prime}(x)+(E-V(x)) u(x)=0 .
$$

Стандартной заменой $\eta=\Psi u, \Psi(x)=\exp \left\{2^{-1} \int V^{\prime}(x)[E-V(x)]^{-1} d x\right\}$, приведем (1) к виду

$$
\eta^{\prime \prime}+\left[(E-V)-\frac{2 V^{\prime \prime}(E-V)+3 V^{\prime 2}}{(E-V)^{2}}\right] \eta=0 .
$$

Сравним (2) с уравнением

$$
\varphi^{\prime \prime}+(E-V) \varphi=0 .
$$

Пусть $\varphi(x)$ - собственная функция задачи Неймана, соответствующая числу $\nu_{k+1}$; тогда $\varphi$ имеет ровно $k+1$ нулей в интервале $(-1,1)$. Таким образом, в интервале $(-1,1)$ функция $\varphi^{\prime}$ имеет не менее $k$ нулей, а на отрезке $[-1,1]$ - не менее $k+2$ нулей. Следовательно, и функция $\eta=\Psi \varphi^{\prime}$, будучи решением уравнения (2), имеет на этом отрезке хотя бы $k+2$ нулей. Пусть $\psi(x)=\psi(x ; E)$ - решение уравнения (3), удовлетворяющее условиям $\psi(-1)=0, \psi^{\prime}(-1)=1$. По теореме Штурма $\psi(x)$ имеет не менее $k+1$ нулей на интервале $(-1,1)$ и, значит, не менее $k+2$ нулей на промежутке $[-1,1)$. Уменьшая $E$ в уравнении $(3)$, мы заставим нули функции $\psi$ двигаться вправо. Когда $(k+2)$-й нуль этой функции (считаем -1 первым нулем) достигнет точки 1 , мы получим решение задачи Дирихле с собственным числом $\mu_{k}$. Значит, $\mu_{k}<\nu_{k+1}$.

Дадим набросок доказательства теоремы, пользуясь введенными выше обозначениями. Не умаляя обшности, будем считать далее, что $U(0)=0$. Выберем систему функций $\Phi_{1}(x ; E), \Phi_{2}(x ; E)$, являющуюся при каждом $E \in \mathbb{R}$ фундаментальной системой решений уравнения $-u^{\prime \prime}+V(x) u=E u$; можно считать, что эти функщии аналитически зависят от $x$ и $E$. Пусть

$$
D(T ; E)=\left|\begin{array}{cc}
\Phi_{1}(-T ; E) & \Phi_{2}(-T ; E) \\
\Phi_{1}(T ; E) & \Phi_{2}(T ; E)
\end{array}\right| .
$$

Заменяя в (4) функции $\Phi_{j}$ их производными по $x$, определяем функцию $N(T ; E)$; аргумент $T$ в обеих функциях лежит на полуоси $T>0$, а $E$ - любое вещественное число. Как известно, числа $\mu_{k}(T)$ (соответственно $\nu_{k}(T)$ ) являются простыми корнями уравнения $D(T ; E)=0(N(T ; E)=0)$ относительно $E$. Легко видеть, что при наших предположениях $\mu_{k}(T), \nu_{k+1}(T) \geqslant(k+1)^{2} \pi^{2} / 4 T^{2}$; поэтому при всех $k \in \mathbb{N}$ справедливы соотношения

$$
\lim _{T \rightarrow 0} \mu_{k}(T)=\lim _{T \rightarrow 0} \nu_{k+1}(T)=+\infty .
$$

Введем двумерные векторы

$$
\boldsymbol{u}_{j}=\left(\Phi_{1}\left(T_{j} ; E\right), \Phi_{2}\left(T_{j} ; E\right)\right), \quad \boldsymbol{u}_{j}^{\prime}=\left(\Phi_{1}^{\prime}\left(T_{j} ; E\right), \Phi_{2}^{\prime}\left(T_{j} ; E\right)\right),
$$


где $j=1,2, T_{1}=-T, T_{2}=T$. Нетрудно доказать такое утверждение:

(I) $E c \wedge u E=\mu_{k}(T)$ илu $E=\nu_{k}(T)$, mo $\boldsymbol{u}_{1} \wedge \boldsymbol{u}_{1}^{\prime}=\boldsymbol{u}_{2} \wedge \boldsymbol{u}_{2}^{\prime} \neq 0$.

Из этого утверждения в свою очередь вытекают следующие:

(II) $\mu_{k}^{\prime}(T)<0$ nри всех $T>0 u k \in \mathbb{N}$.

(III) Пусть $k \geqslant 1$. Тогда существует единственная точка $T_{k}^{0}>0$, являюшаяся решением уравнения

$$
V(T)=\nu_{k}(T) ;
$$

при этом $\nu_{k}^{\prime}(T)<0$, если $T<T_{k}^{0}, u \nu_{k}^{\prime}(T)>0$, если $T>T_{k}^{0}$.

Для доказательства утверждения (II) заметим, что из (I) вытекает соотношение $\partial D(T ; E) / \partial T \neq 0$ при $E=\mu_{k}(T)$. Отсюда $\mu_{k}^{\prime}(T) \neq 0$ при всех $T$, и осталось сослаться на (5).

Для доказательства (III) воспользуемся равенством

$$
\partial N(T ; E) / \partial T=(V(T)-E)\left(\boldsymbol{u}_{1} \wedge \boldsymbol{u}_{2}^{\prime}-\boldsymbol{u}_{1}^{\prime} \wedge \boldsymbol{u}_{2}\right),
$$

с учетом которого из (I) получаем, что уравнение (6) равносильно уравнению $\nu_{k}^{\prime}(T)=0$. Если бы уравнение (6) не имело корней, то из (5) получилось бы неравенство $\nu_{k}^{\prime}(T)<0$ для любого $T>0$. С другой стороны, $V(T)$ неограниченно возрастает при $T>0$; поэтому из (5) получаем, что графики функций $V$ и $\nu_{k}$ пересекаются; противоречие. В силу аналитичности рассматриваемых функций на полуоси $T>0$ существует наименьший корень уравнения (6); обозначим его через $T_{k}^{0}$. Допустим, что это уравнение имеет еще хотя бы один корень, и пусть $T^{\prime}$ - наименьший из них. Несложные рассуждения показывают, что тогда $V^{\prime}\left(T^{\prime}\right) \leqslant 0$, а это противоречит свойствам функции $V$. Учитывая (5), для завершения доказательства утверждения (III) достаточно показать, что $\nu_{k}^{\prime \prime}\left(T_{k}^{0}\right) \neq 0$. Это будет доказано, если мы покажем, что $\partial^{2} N / \partial T^{2} \neq 0$ при $T=T_{k}^{0}, E=\nu_{k}\left(T_{k}^{0}\right)$. Если это не так, то при тех же значениях $T$ и $E$ прямым подсчетом приходим к равенству $V^{\prime}(T)\left(\boldsymbol{u}_{1} \wedge \boldsymbol{u}_{2}^{\prime}-\boldsymbol{u}_{1}^{\prime} \wedge \boldsymbol{u}_{2}\right)=0$, которое противоречит утверждению (I).

Теперь легко завершить доказательство теоремы. Пусть сначала $0<T<$ $T_{k+1}^{0}$. Тогда в силу (III) $V(T)<V\left(T_{k+1}^{0}\right)=\nu_{k+1}\left(T_{k+1}^{0}\right)<\nu_{k+1}(T)$; поэтому из леммы вытекает, что $\mu_{k}(T)<\nu_{k+1}(T)$. В пределе имеем $\mu_{k}\left(T_{k+1}^{0}\right) \leqslant \nu_{k+1}\left(T_{k+1}^{0}\right)$. Пусть теперь $T>T_{k+1}^{0}$. Тогда в силу (II) и (III) $\nu_{k+1}(T)>\nu_{k+1}\left(T_{k+1}^{0}\right) \geqslant$ $\mu_{k}\left(T_{k+1}^{0}\right)>\mu_{k}(T)$. Осталось убедиться, что $\mu_{k}\left(T_{k+1}^{0}\right) \neq \nu_{k+1}\left(T_{k+1}^{0}\right)$. Но в противном случае из равенств $\mu_{k}\left(T_{k+1}^{0}\right)=\nu_{k+1}\left(T_{k+1}^{0}\right)$ и $\nu_{k+1}^{\prime}\left(T_{k+1}^{0}\right)=0$ и уже доказанных неравенств легко получить, что $\mu_{k}^{\prime}\left(T_{k+1}^{0}\right)=0$, а это противоречит (II). Теорема доказана.

ЗАмЕчАНИЕ. В силу четности потенциала $U$ мы имеем на самом деле $\mu_{k}=$ $\lambda_{k+1}, \nu_{k+1}=\widetilde{\lambda}_{k+1}$. В [4] доказано, что в подпространстве $E_{0} \subset L^{2}(-T, T)$ четных $2 T$-периодических потенциалов $U$ с нулевым средним на отрезке $[-T, T]$ соответствие $U \mapsto\left(\nu_{k+1}-\mu_{k}\right)_{k \geqslant 1}$ есть вещественно-аналитический изоморфизм пространства $E_{0}$ на пространство $l^{2}$. Результаты заметки дают примеры потенциалов, образы которых при этом изоморфизме лежат в конусе положительных элементов $l_{+}^{2}$. Отметим также, что в отличие от $[3,4]$ результаты нашей заметки не позволяют получить потенциал по заранее заданному набору спектральных данных. 
Наконец, заметим, что к доказательству неравенств $\mu_{k}(T)<\nu_{k+1}(T)$ сводится строгое обоснование результатов об отсутствии рассеяния назад краевых состояний в квантовом эФФекте Холла [6]; в ряде работ эти результаты получались численными методами.

В заключение авторы благодарят рецензента за замечания, способствовавшие улучшению рукописи.

\title{
ЛитеРАтУРА
}

1. Рид М., Саймон Б. Методы современной математической физики, т. 4. Мир, М., 1982 . 2. Станкевич И. В. ДАН СССР, 192, №1, 34-37 (1970). 3. Марченко В. А., Островский И. В. Матем. сб., 97, вып. 4, 540-606 (1975). 4. Garnett J., Trubowitz E. Comment. Math. Helv., 59, 258-315 (1984). 5. Левитан Б. М., Сарәсян И. С. Операторы Штурма-Лиувилля и Дирака. Наука, М., 1988. 6. Квантовый эффект Холла. Сб. статей (Сост. А. Я. Шик, Ю. В. Шмарцев), Мир, М., 1986.

Мордовский государственный университет им. Н. П. Огарёва
Поступило в редакцию 20 февраля 1996 г.

\section{Спектральный анализ задачи Редже с параметрами}

\author{
(c) 1997. Г. М. ГуБРеЕв, В. Н. ПивовАРЧИк
}

Заметка посвящена исследованию спектральной задачи

$$
\begin{gathered}
-y^{\prime \prime}+q(x) y=\lambda^{2} y, \\
y(0)=0, \quad y^{\prime}(a)+(i \alpha \lambda+\beta) y(a)=0, \quad \alpha>0, \beta \in \mathbb{R},
\end{gathered}
$$

с вещественным потенциалом $q \in L_{2}(0, a)$ и параметрами $\alpha, \beta$. Введение параметров объясняется тем, что к такой задаче с помощью преобразования Лиувилля приводится задача о колебаниях гладкой струны с вязким трением на правом конще, причем ограничение $\alpha>0$ вызвано тем, что этот параметр пропорционален коэффициенту трения. При $\alpha=1, \beta=0$ получаем классическую задачу Редже [1], возникающую при описании рассеяния на финитном потенциале. В [1] для случая однократного спектра доказана полнота системы собственных функций задачи в пространстве $L_{2}(0,2 a)$ в предположении, что $a \in \operatorname{supp} q$. Отсюда выводится (кстати, некорректно) возможность разложения в ряды по собственным функциям задачи произвольной функции из $L_{2}(0,2 a)$. Опираясь на эти разложения, автор дает решение интегрального уравнения ГельфандаЛевитана, которое позволяет восстановить потенциал по заданной матрице рассеяния.

Настоящая заметка посвящена развитию этих исследований. Оставляя в стороне общий случай, объясним суть этого развития на примере задачи Редже $(\alpha=1, \beta=0)$. Здесь впервые дано полное описание спектра такой задачи

* Эта работа выполнена при поддержке Международного научного фонда, гранты $\mathrm{UCZ000} \mathrm{и} \mathrm{UCZ200.}$ 